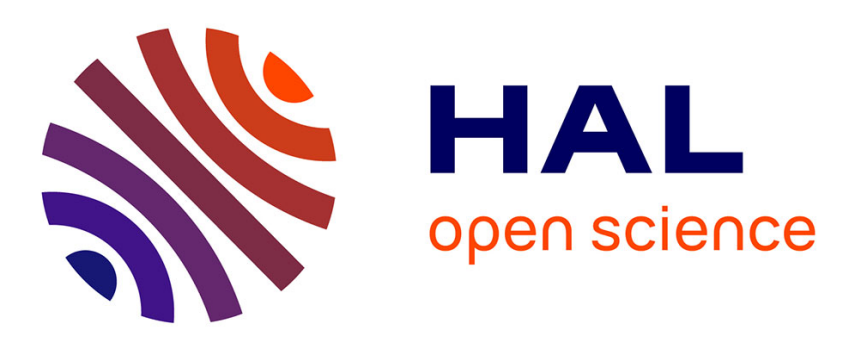

\title{
Mechanical Stop Mechanism For Overcoming MEMS Fabrication Tolerances
}

Hussein Hussein, Gilles Bourbon, Patrice Le Moal, Yassine Haddab, Philippe Lutz

\section{- To cite this version:}

Hussein Hussein, Gilles Bourbon, Patrice Le Moal, Yassine Haddab, Philippe Lutz. Mechanical Stop Mechanism For Overcoming MEMS Fabrication Tolerances. Journal of Micromechanics and Microengineering, 2017, 27 (1), pp.017001. 10.1088/0960-1317/27/1/017001 . lirmm-02006512

\section{HAL Id: lirmm-02006512 https://hal-lirmm.ccsd.cnrs.fr/lirmm-02006512}

Submitted on 4 Feb 2019

HAL is a multi-disciplinary open access archive for the deposit and dissemination of scientific research documents, whether they are published or not. The documents may come from teaching and research institutions in France or abroad, or from public or private research centers.
L'archive ouverte pluridisciplinaire HAL, est destinée au dépôt et à la diffusion de documents scientifiques de niveau recherche, publiés ou non, émanant des établissements d'enseignement et de recherche français ou étrangers, des laboratoires publics ou privés. 


\title{
Mechanical Stop Mechanism For Overcoming
} MEMS Fabrication Tolerances

Keywords: Mechanical stop mechanism, fabrication tolerances, discrete positioning, MEMS

Submitted to: J. Micromech. Microeng. Yassine Haddab ${ }^{2}$ and Philippe Lutz ${ }^{1}$

${ }^{1}$ Femto-st Institute, Besancon, France

${ }^{2}$ Lirmm laboratory, Montpellier, France

E-mail: hussein.hussein@femto-st.fr

August 2016

\begin{abstract}
A mechanical stop mechanism is developed in order to compensate MEMS fabrication tolerances in discrete positioning. The mechanical stop mechanism is designed to be implemented on SOI wafers using a common DRIE etching process. The various fabrication tolerances obtained due to the etching process are presented and discussed in the paper. The principle and design of the mechanism are then presented. Finally, experiments on microfabricated positioning prototypes show accurate steps unaffected by the fabrication tolerances.
\end{abstract}




\section{Introduction}

Discrete positioning MEMS, such as switches [1, 2], valves [3], relays [4], positioners [5], braille displays [6], and digital microrobots $[7,8,9,10,11,12,13]$ have limited number of well defined repeatable states called "discrete positions".

The discrete positioning concept has many advantages in MEMS, it allows robust and repeatable positions without feedback. No sensor is needed which simplifies the structure and the integration of the system in complicated environments. This allows going further in miniaturization and avoiding the general problems of sensors in microstructures (size, connectivity, noise accuracy, control laws, etc.).

The positioning accuracy and dimensions tolerances are the key tools in the performance characterization of discrete positioning MEMS. However, the design of these systems is still suffering from the fabrication tolerances where the positioning steps and dimensions after fabrication show unexpected differences from the design.

In the other side, the monolithic structure is an essential characteristic in standard MEMS-based fabrication processes. The structure in these processes is fabricated by etching deeply a flat surface. The components in the as-fabricated configuration are free without residual constraints. This means that the moving part in a discrete positioning MEMS cannot be directly fabricated in a discrete position, which is maintained by other components. Otherwise, the moving part will be fixed to these components. The solution in this case is usually to attach the moving part manually after fabrication in face of a mechanical stop and use its stiffness in order to maintain the position (e.g., stop blocks in [14]).

A mechanical stop mechanism is presented in this paper. The manual activation of this mechanism after fabrication allows inserting the shuttle of a discrete positioning MEMS between two mechanical stops. This results in three positions of the shuttle: as-fabricated position, and 2 discrete positions in face of the mechanical stops. The advantage of this mechanism is that the distances between the three positions are independent from the fabrication tolerances.

In the following, usual fabrication tolerances are introduced and discussed. The principle and design of the mechanical stop mechanism are described. Finally, the experiments on fabricated prototypes of the mechanism are presented.

\section{Microfabrication tolerances}

Selection of the fabrication process depends upon the specific application, material, tolerance, size of features and aspect ratio. The fabrication tolerances in this paper concern devices that are realized using a classical bulk micromachining of a single-crystalline silicon substrate.

Single-crystalline silicon material is commonly used in a wide variety of MEMS and has many important properties such as an almost perfect elastic behavior with highly repeatable motion and without hysteresis and energy dissipation, long lifetime with little fatigue, low cost and ability to incorporate electronic functionality such as the electrothermal actuators.

Many parameters affect the patterns final form and the resulting tolerances in each step of fabrication. Figure 1 shows the main steps for etching the device layer.

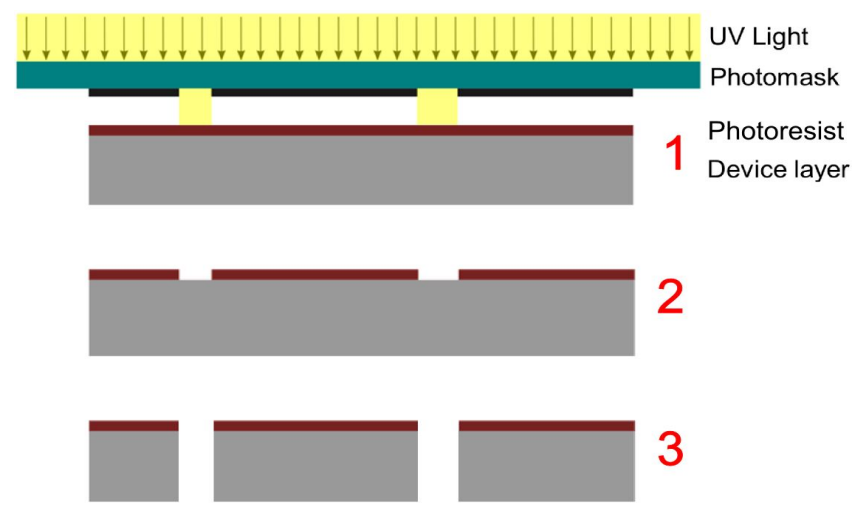

Figure 1. Etching process. Photoresist deposition and UV light exposure using a photomask (1), photoresist developing (2), DRIE of the silicon layer (3).

Manufacturing the photomask involves unavoidable tolerances. The photomask is a patterned chromium coated glass, the pattern information is created in a cad software and transferred to the photomask using laser or e-beam writer. The patterns in the photomask shows some differences from the design due to the influence of some parameters (laser or electron density, etcher concentration, etching time of the chromium layer, etc.).

In the first step (Figure 1), the wafer is covered with positive photoresist layer by spin coating, then the photoresist is exposed to a pattern of UV light through the photomask.

In the second step, the imaged patterns of the photoresist on the device layer are developed. The tolerances in this step are related to several parameters (photoresist quality, developing time, developer concentration, bake recipes, etc.).

In the third step, the silicon is etched using DRIE, which is one of the most popular fabrication techniques for silicon bulk micromachining. DRIE is characterized by a high etch rate, high selectivity to silicon dioxide 
and etching masks and a flexible transition between anisotropic and isotropic etching with the objective of realizing high aspect ratio microstructures and vertical sidewalls.

DRIE is done using Bosch process with alternating passivation $\left(C_{4} F_{8}\right)$ and etching $\left(S F_{6}\right)$ steps. However, this fabrication technique induces various fabrication tolerances such as microloading effect $[15,16]$, notching or footing effect [17], lag effect in the small openings [18], slanted profiles and undercut [19, 20].

These tolerances may affect the mechanical stiffness, displacement, performance of devices in MEMS and would induce a mismatching between the measured dimensions and the designed values.

The various tolerances are dependent on the process parameters (gas flowrate, power, pressure, temperature, cycling time, etc.) but also on the feature sizes. In fact, the etching tolerances evolve with the width of the openings $[15,16,17,18,19,20]$.

In order to obtain a uniform and homogeneous etching throughout the photomask, the photoresist and the wafer, the silicon layers are etched with a unified opening width in the fabrication process. The fabrication on a wafer with exactly the same parameters and the same opening width leads necessarily to the same form and dimensions of the fabrication tolerances throughout the sidewalls of the patterns, mainly in a local zone of the wafer.

Based on this consideration, gain or loss in the sidewalls dimensions have the same value throughout a microdevice that is locally fabricated on a wafer.

Noting that the layout to be etched contains parts to be released from the device during the fabrication process. The surrounding of these parts is etched which separate them from the device. The existence of these parts make it possible to etch with the same opening width all over the wafer.

Figure 2 shows the variations in width and distance between the sidewalls of two parallel patterns in the design and after fabrication. $\Delta$ is the value of gain or loss in each sidewall dimension after fabrication. Positive values of $\Delta$ are considered when there is a loss in the width of patterns.

As shown in figure 2, due to the uncertainty of dimensions after fabrication, $2 \Delta$ is added to the distance between two faced sidewalls $(d \rightarrow d+2 \Delta)$ and is subtracted from the distance between two opposite sidewalls $\left(d+w_{1}+w_{2} \rightarrow d+w_{1}+w_{2}-2 \Delta\right)$. Another important feature is that the distance between two sidewalls from the same side (right or left) remains the same after fabrication $\left(d+w_{1} \rightarrow d+w_{1}\right)$.

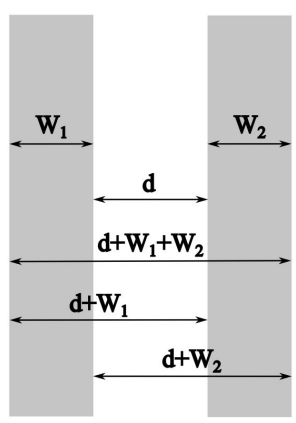

(a)

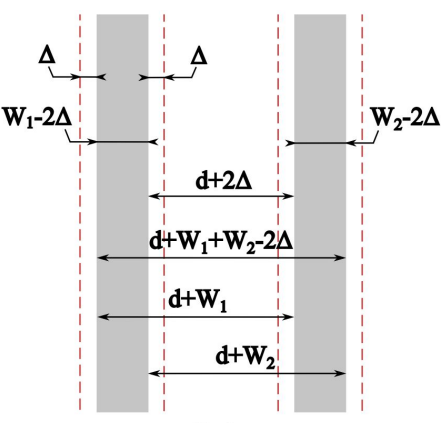

(b)
Figure 2. Distances between the sidewalls of two parallel patterns in the design (a) and after fabrication (b).

\section{Mechanical stop mechanism}

In the design of the mechanical stop mechanism, we take advantage of the effect that the distance between two parallel patterns sidewalls in the design increases and decreases after fabrication according to the different sidewalls.

The mechanical stop mechanism is designed in order to insert a shuttle with one axis displacement between two mechanical stops. These stops ensure an accurate stroke $s$ between two discrete positions of the shuttle and an accurate activation distance $d$ between the shuttle in its as-fabricated position and its first discrete position. The values of $s$ and $d$ are independent from the fabrication tolerances.

Figure 3 shows a drawing of a positioning device where a mechanical stop mechanism is used to define two discrete positions. The shuttle is guided vertically with four curved beams and can be switched using electrothermal actuators.

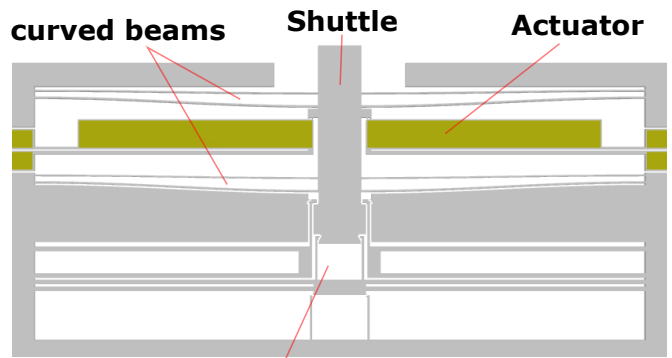

Accurate positioning mechanism

Figure 3. Positioning device using a mechanical stop mechanism.

Figure 4.a shows the components of the mechanical stop mechanism, it consists of lower and upper stops and locks and the shuttle. The important distances $d_{1}, d_{2}, d_{3}$ and $d_{4}$ between the different components are shown with considering the fabrication tolerances.

The lower and upper stops are designed to be suspended to the lower and upper locks respectively in 


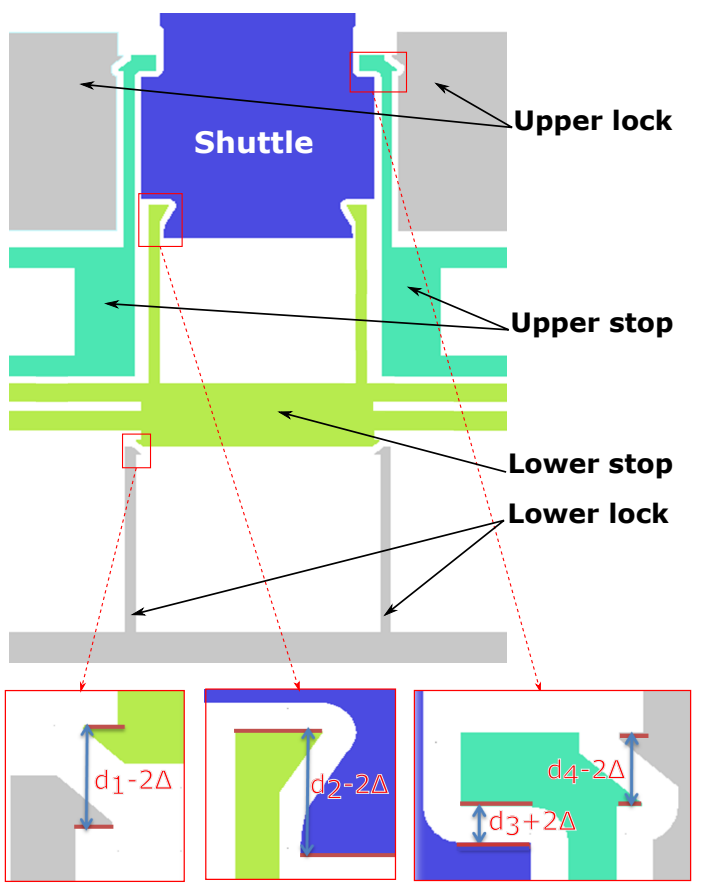

(a)

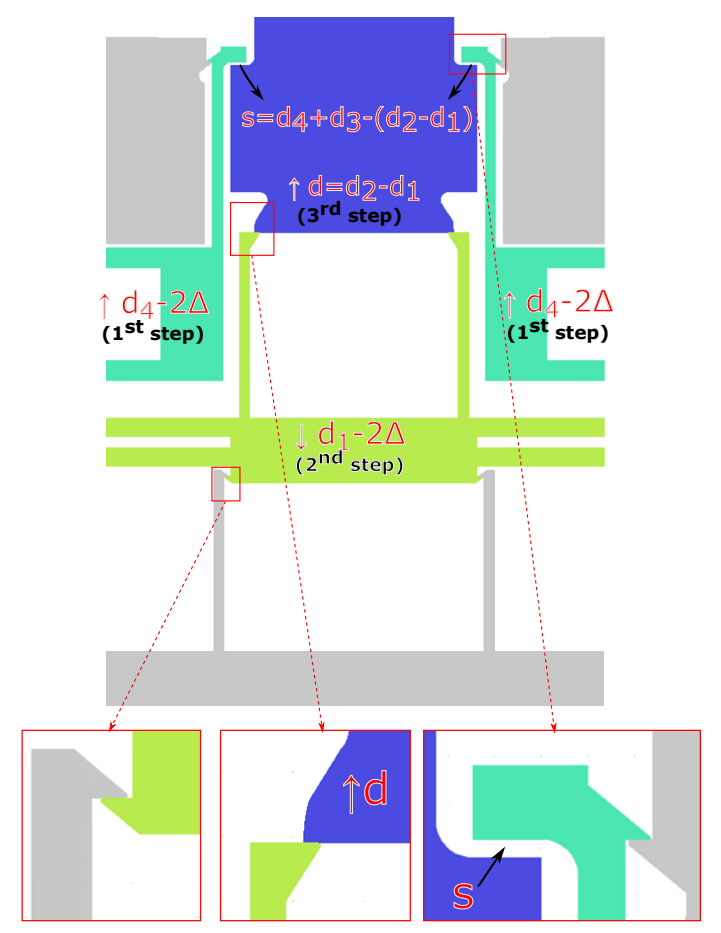

(b)

Figure 4. Drawing of the different components in the mechanical stop mechanism and the important distances between the different components (a). Drawing of the mechanical stop mechanism after activation where the movable parts are suspended to their locks and the shuttle is in the initial position (b).

the activation phase. These components are suspended using the triangular-shaped head which allows sliding from a side and blocking displacement from the other side. The deformable beams in the stops and lower locks are used due to their transversal flexibility during suspension of the different components.

The activation phase is a sequence of several steps which are made manually after fabrication, once for all. In the first step, the upper stop is suspended to the upper lock by moving it upwards a distance $d_{4}-2 \Delta$. In the second step, the lower stop is suspended to the lower locks by moving it downwards a distance $d_{1}-2 \Delta$. In the third step, the shuttle is pushed beyond the head of the deformable beams in the lower stop. Its position is then limited between the lower and upper stops. Figure 4.b shows the mechanical stop mechanism after activation with the different distances.

The stiffness of the curved beams ensures that the shuttle remains in contact with the lower stop (first discrete position). The second discrete position is when the shuttle becomes in contact with the upper stops.

As shown in figure 4.b, the initial displacement $d$ of the shuttle and the stroke $s$ between the two discrete positions are independent from the fabrication tolerances:

$$
\begin{aligned}
& d=d_{2}-d_{1} \\
& s=d_{4}+d_{3}-\left(d_{2}-d_{1}\right)
\end{aligned}
$$

In result, the mechanical stop mechanism compensates the fabrication tolerances, defines accurately the stroke between the 2 discrete positions and places the shuttle in its first position where the stiffness of the curved beams holds the shuttle.

\section{Experiments on positioning devices}

Some prototypes of the positioning device with mechanical stop mechanisms were fabricated on SOI wafers $(100 \mu m$ device layer, a $2 \mu m$ buried oxide and a $380 \mu m$ handle layer). The width of the openings in the device layer are unified to $20 \mu \mathrm{m}$ in the fabrication process in order to ensure homogeneous etching conditions throughout the wafer.

The internal dimensions of these prototypes are equivalent to $7 \mathrm{~mm} \times 2.9 \mathrm{~mm}$. The mechanical stop mechanism allows defining any value for the stroke $s$. It is chosen to be equivalent to $10 \mu \mathrm{m}$ in the prototypes. The distance $d_{3}$ is equivalent to the unified opening width in the device layer (i.e. $d_{3}=20 \mu \mathrm{m}$ ). The distances $d_{1}$ and $d_{4}$ must contain 2 triangular heads which are separated by the width of the opening between them. $d_{1}$ and $d_{4}$ are chosen to be equivalent to $40 \mu \mathrm{m}$ in the prototype. Higher values of $d_{1}$ and $d_{4}$ allow higher sizes of the triangular heads which increases and enhances the contact surface between them. The distances $d$ and $d_{2}$ are then calculated from (1) $\left(d=50 \mu \mathrm{m}\right.$ and $\left.d_{2}=90 \mu \mathrm{m}\right)$. 
After suspending the stops to their activated positions, the displacement of the stops in the backward directions is constrained by the locks. As for the other direction, due to the stiffness of the elastic beams that relate the stops to the outer support, the lower stop remains stable in the suspended position without displacement unless the applied forces exceed $14.5 \mathrm{mN}$ in the downward direction, and the upper stops remain stable in the suspended position unless the applied forces exceed $15 \mathrm{mN}$ in the upward direction. The value of these retraction forces can be changed with respect to the elastic beams dimensions.

The curved beams, which relate the shuttle to the outer support, are used due to the specific evolution of their snapping forces, which start to decrease after a certain limit of displacement [21]. Thereby, the dimensions of the curved beams are chosen to ensure a significant holding force in the first position and to avoid high loads on the actuators while switching to the second position.

The snapping force of the curved beams used in the prototypes is equivalent to $4.4 \mathrm{mN}$ at the first position $(50 \mu \mathrm{m})$ and to $4.15 \mathrm{mN}$ at the second position $(60 \mu \mathrm{m})$. In contrast to the positioning, the values of the different forces is still dependent from the fabrication tolerances.

After fabrication, the activation phase is made manually as explained previously using the needle of a Probehead PH100.

Afterwards, the experiments were made on several prototypes by supplying the actuators by voltage steps through the needles of the probeheads that are placed on the gold pads.

Prototypes were fabricated on several wafers and distributed in different places in the wafer. It was remarked that the fabrication tolerances were different from a wafer to the other. For example, the tolerance per edge on the photomask was equivalent to $0.2 \mu \mathrm{m}$. The tolerance per edge on the developed photoresist before etching was equivalent to $0.8 \mu \mathrm{m}$ on a wafer and to $1.1 \mu \mathrm{m}$ on another wafer. Finally, the tolerance per edge of silicon patterns after DRIE was equivalent to $1.4 \mu \mathrm{m}$ on a wafer and to $1.8 \mu \mathrm{m}$ on another wafer. Those various tolerances were compensated on all the tested devices as measured using a specific camera that is mounted on the top of an optical microscope.

Figure 6 shows a positioning device prototype before and after switching the shuttle between the first and second positions. The switching time is about $20 \mathrm{~ms}$ after applying a voltage of $18 \mathrm{~V}$ on the actuators.

The experiments show that the positioning device

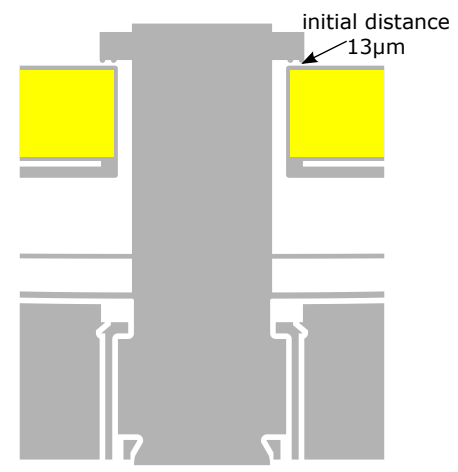

(a)

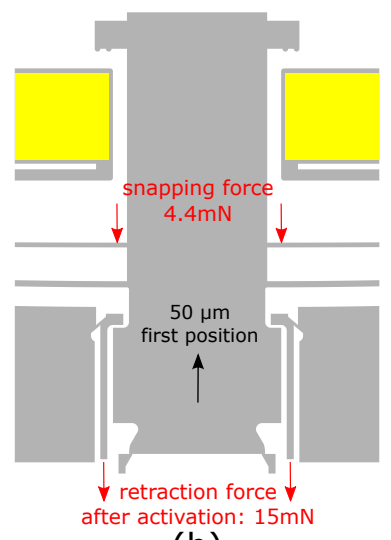

(b)

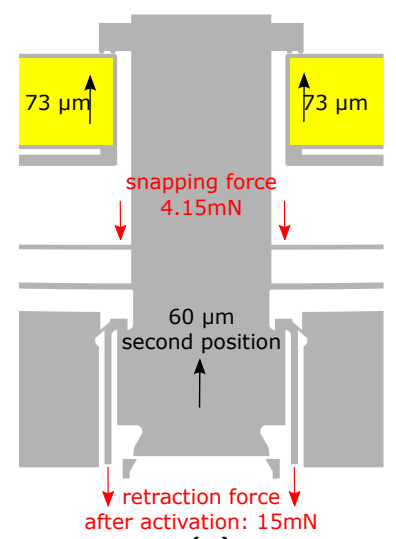

(c)
Figure 5. Important distances and elastic forces in the asfabricated configuration of the positioning device (a), after activation (first position) (b) and after switching to the second position (second position) (c).

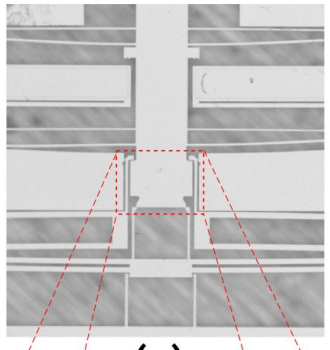

(a)

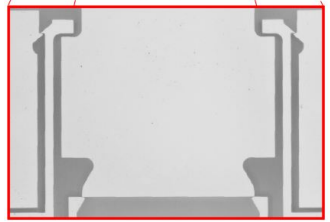

(c)

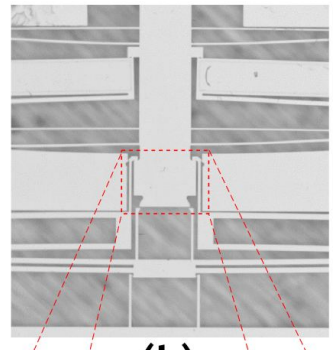

(b)

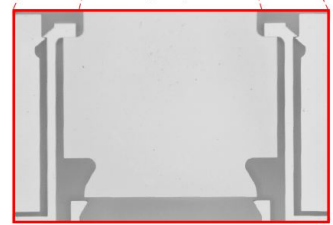

(d)
Figure 6. Functioning of the positioning device in the experiments. (a) and (b) show the positioning device in the first and second positions respectively. (c) and (d) shows a zoom on the mechanical stop mechanism in the two positions respectively.

works properly and the measurements using the camera show that the displacement of the shuttle (i.e. $d$ and $s$ ) is independent from the fabrication 
tolerances. The mechanical stop mechanism combines the advantages of discrete positioning, monolithic and compliant structures. Its simplicity allows it to be used in more complex systems for accurate switching applications and more advanced tasks. The design dimensions can be adjusted as required for each application.

\section{Conclusion}

In this paper, we have presented a new concept of a mechanical stop mechanism that compensates the tolerances resulting from the fabrication process and defines accurately the activation distance and displacement stroke between two discrete positions.

The principle and design of the mechanical stop mechanism and the different components were presented. Some prototypes of a positioning device with mechanical stop mechanism were fabricated on SOI wafers and the experiments showed accurate positioning without the influence of the fabrication tolerances.

\section{Acknowledgment}

This work has been supported by the Labex ACTION project (contract "ANR-11-LABX-01-01"), the French RENATECH network and its FEMTO-ST technological facility.

\section{References}

[1] Gabriel M Rebeiz and Jeremy B Muldavin. Rf mems switches and switch circuits. Microwave Magazine, IEEE, 2(4):59-71, 2001.

[2] Peter De Dobbelaere, Ken Falta, Steffen Gloeckner, and S Patra. Digital mems for optical switching. Communications Magazine, IEEE, 40(3):88-95, 2002.

[3] Peter Galambos, Jeffrey Lantz, Michael S Baker, Jaime McClain, Gregory R Bogart, and Robert Joseph Simonson. Active mems valves for flow control in a highpressure micro-gas-analyzer. Microelectromechanical Systems, Journal of, 20(5):1150-1162, 2011.

[4] Yongfeng Gao, Zheng You, and Jiahao Zhao. Electrostatic comb-drive actuator for mems relays/switches with double-tilt comb fingers and tilted parallelogram beams. Journal of Micromechanics and Microengineering, 25(4):045003, 2015.

[5] Guimin Chen, Yanjie Gou, and Aimei Zhang. Synthesis of compliant multistable mechanisms through use of a single bistable mechanism. J. Mech. Des., 133(8):081007-081007, August 2011.

[6] Xiaofan Niu, Paul Brochu, Hristiyan Stoyanov, Sung Ryul Yun, and Qibing Pei. Bistable electroactive polymer for refreshable braille display with improved actuation stability. In Proc. of SPIE Vol, volume 8340, page 83400R1, 2012.

[7] Qiao Chen, Yassine Haddab, and P. Lutz. Digital microrobotics based on bistable modules: Design of compliant bistable structures. In IEEE/ASME International Conference on Mechtronic and Embedded
Systems and Applications, 2008. MESA 2008, page 3641, 2008.

[8] Paul Cazottes, A Fernandes, Joël Pouget, and Moustapha Hafez. Actuation of bistable buckled beams with macrofiber composites. In Intelligent Robots and Systems, 2008. IROS 2008. IEEE/RSJ International Conference on, pages 564-569. IEEE, 2008.

[9] Seunghoon Park and Dooyoung Hah. Pre-shaped buckledbeam actuators: theory and experiments. Sensors and Actuators A: Physical, 148(1):186-192, 2008.

[10] Vincent Chalvet, Yassine Haddab, and Philippe Lutz. A microfabricated planar digital microrobot for precise positioning based on bistable modules. Robotics, IEEE Transactions on, 29(3):641-649, 2013.

[11] Laurent Petit. Contribution aux techniques d'actionnement numérique : cas d'un système électromagntique $2 D$. $\mathrm{PhD}$ thesis, Université de technologie de Compiégne, 2009.

[12] Dung-An Wang, Huy-Tuan Pham, and Yi-Han Hsieh. Dynamical switching of an electromagnetically driven compliant bistable mechanism. Sensors and Actuators A: Physical, 149(1):143-151, 2009.

[13] HUYAN Pengfei. Electromagnetic digital actuators array: Characterization of a planar conveyance application and optimized design. PhD thesis, Université de technologie de Compiégne, 2015.

[14] Qiao Chen. Microrobotique numérique fondée sur l'utilisation de modules bistables : conception, fabrication et commande de modules monolithiques. phdthesis, Université de Franche-Comté, 2010.

[15] C Hedlund, H-O Blom, and S Berg. Microloading effect in reactive ion etching. Journal of Vacuum Science 8 Technology A, 12(4):1962-1965, 1994.

[16] Junghoon Yeom, Yan Wu, and Mark A Shannon. Critical aspect ratio dependence in deep reactive ion etching of silicon. In TRANSDUCERS, SolidState Sensors, Actuators and Microsystems, 12th International Conference on, 2003, volume 2, pages 1631-1634. IEEE, 2003.

[17] Gyeong S Hwang and Konstantinos P Giapis. On the origin of the notching effect during etching in uniform high density plasmas. Journal of Vacuum Science 86 Technology B, 15(1):70-87, 1997.

[18] Yiyong Tan, Rongchun Zhou, Haixia Zhang, Guizhang Lu, and Zhihong Li. Modeling and simulation of the lag effect in a deep reactive ion etching process. Journal of Micromechanics and Microengineering, 16(12):2570, 2006.

[19] J Li, AQ Liu, and QX Zhang. Tolerance analysis for comb-drive actuator using drie fabrication. Sensors and Actuators A: Physical, 125(2):494-503, 2006.

[20] Sergi Gomez, Rodolfo Jun Belen, Mark Kiehlbauch, and Eray S Aydil. Etching of high aspect ratio structures in si using sf 6/o 2 plasma. Journal of Vacuum Science 83 Technology A: Vacuum, Surfaces, and Films, 22(3):606$615,2004$.

[21] Hussein Hussein, Patrice Le Moal, Gilles Bourbon, Yassine Haddab, and Philippe Lutz. Modeling and stress analysis of a pre-shaped curved beam: Influence of high modes of buckling. International Journal of Applied Mechanics, 7(04):1550055, 2015. 